\title{
Traumatische Dislokation von Os lunatum und Os scaphoideum
}

Luxationsverletzungen des Karpus ohne Frakturen sind seltene Ereignisse. Ursächlich sind erhebliche axiale Gewalteinwirkungen gegen die extendierte Hand, z. B. nach einem Sturz aus großer Höhe oder einem Verkehrsunfall. Häufig findet sich eine perilunäre Luxation in Kombination mit Frakturen des Os scaphoideum (DeQuervainsche Luxationsfraktur), des Os capitatum und des Os triquetrum. Seltener sind isolierte Luxationen ohne knöcherne Beteiligungen. Von besonderem klinischen Interesse sind avaskuläre Nekrosen und begleiten- de Bandverletzungen, deren Defektheilung zur karpalen Instabilität führen können. Eine frühzeitige Reposition ggf. mit operativer Freilegung ist unabdingbare Voraussetzung für eine erfolgreiche Behandlung. Vorgestellt wird eine ungewöhnliche Luxationsverletzung von Os lunatum und Os scaphoideum.

\section{Fallbericht}

Ein 27-jähriger Arbeiter stürzte von einem Baugerüst aus 7 Meter Höhe. Der Sturz wurde durch den wiederholten 
a, b

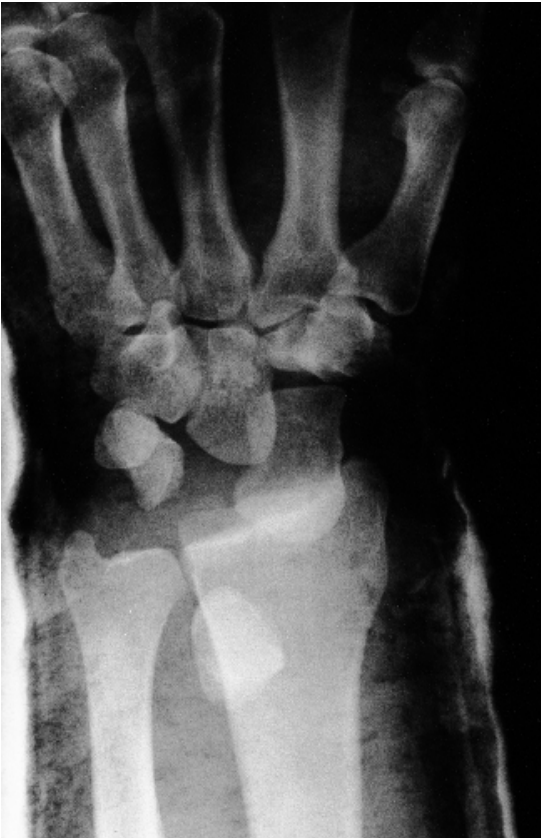

a, b

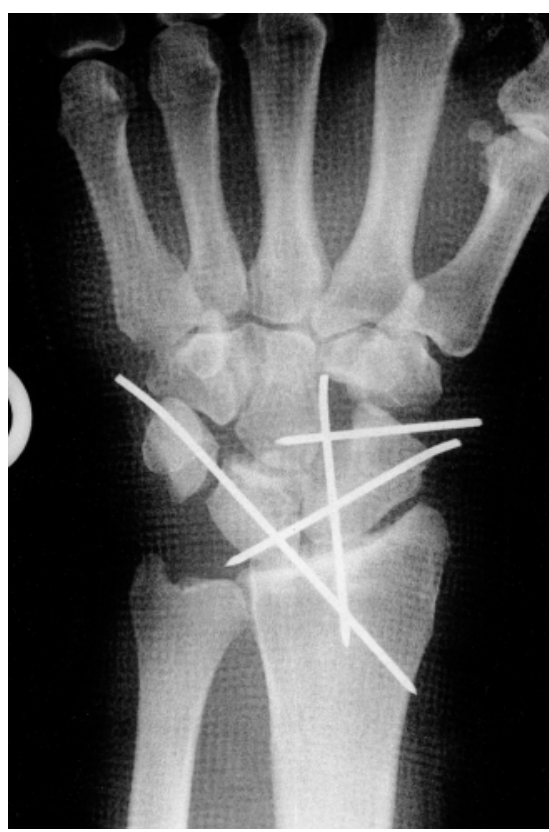

Anprall an das Gerüst abgebremst. Die Hand und das Handgelenk links waren erheblich geschwollen. Bei deutlicher Krepitation bestand der klinische Verdacht einer Luxationsfraktur des Radiokarpalgelenkes. Konventionell radiologisch fand sich eine kombinierte Luxation der Os lunatum und des Os scaphoideum (Abb.1 a). Das Os lunatum war ca. $5 \mathrm{~mm}$ proximal des Radiokarpalgelenkes um seine proximale Gelenkfläche in die palmaren Weichteile des Unterarmes rotiert. Das Os scaphoideum war aus seiner radialen Position gelöst und nach palmar bzw. ulnar verlagert.

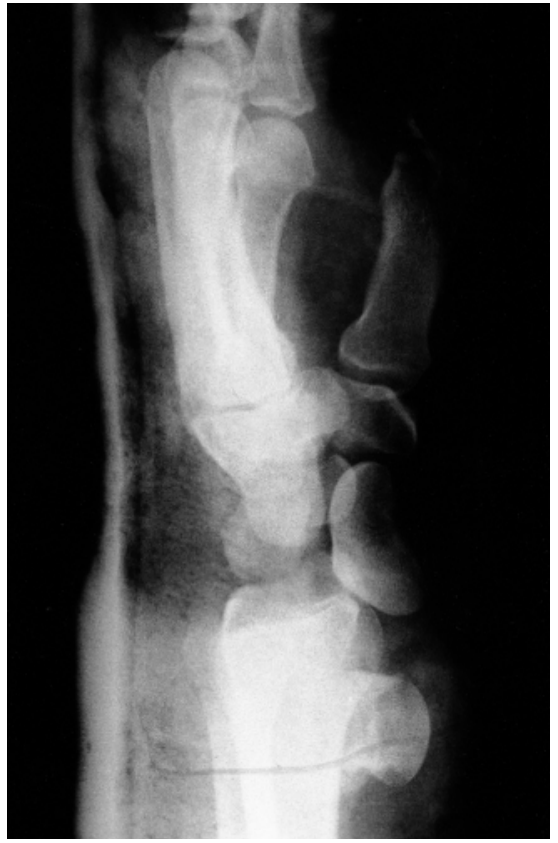

Abb. 1 Konventionell radiologische Untersuchung des linken Handgelenkes (a) im postero-anterioren und (b) im lateralen Strahlengang (Gipsaufnahme). Das Os lunatum ist proximal des Radiokarpalgelenkes in die palmaren Weichteile des Unterarmes rotiert. Das Os scaphoideum ist nach palmar bzw. ulnar verlagert.

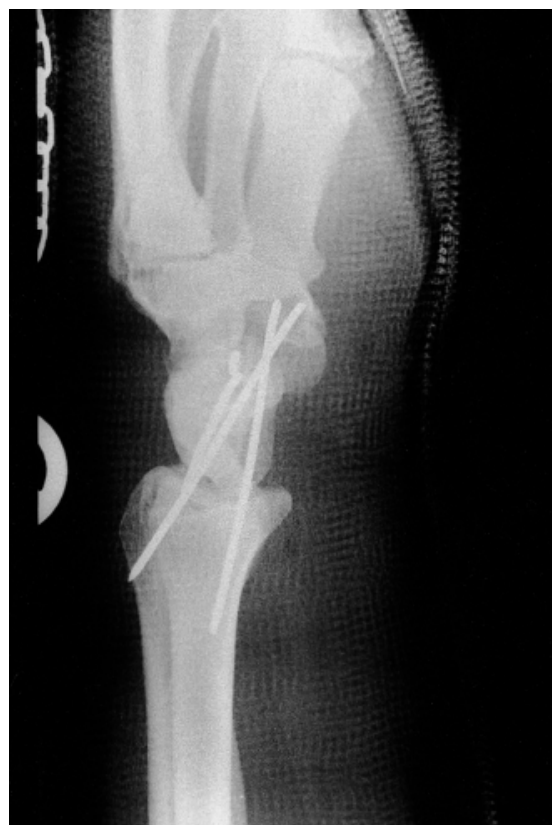

Abb. 2 Konventionell radiologische Untersuchung des linken Handgelenkes (a) im postero-anterioren und (b) im lateralen Strahlengang (Gipsaufnahme). Die Luxationsverletzung wurde offen reponiert und mit Kirschnerdrähten gehalten.

Knöcherne Verletzungen waren nicht nachweisbar.

Da eine geschlossene Reposition nicht möglich war, wurde unmittelbar über einen palmaren Zugang eine operative Einrichtung durchgeführt. Das Os lunatum wurde in einem bis zum Karpaltunnel reichenden Hämatom dargestellt. Während die Ligamenta radiolunata zerrissen waren, gelang es, das Ligamentum ulnolunatum, welches um das Os lunatum rotiert war, mit einem kleinen Gefäßstiel zu erhalten. Das Os scaphoideum konnte geschlossen reponiert wer- den. Das Repositionsergebnis wurde mit 4 Kirschnerdrähten gehalten (Abb. 1 b), die dargestellten Bandstrukturen durch Nähte adaptiert und die transartikuläre Drahtfixation mit einer dorsalen Gipsschiene gesichert.

Eine postoperativ durchgeführte CT der Handwurzel zeigte eine korrespondierende Ausrichtung der Gelenkflächen. Nach 6-wöchiger Gipsbehandlung wurden die Kirschnerdrähte entfernt. Eine ligamentäre Instabilität konnte weder in den klinischen noch in den konventionell radiologischen Verlaufskontrollen nach- 
gewiesen werden. Bei einem guten funktionellen Ergebnis fanden sich auch 4 Monate später keine Hinweise für eine ischämische Nekrose (Abb. 2).

\section{Diskussion}

Eine Dislokation des Os lunatum und des Os scaphoideum ohne knöcherne Beteiligung ist ein seltenes Verletzungsmuster, das in der Literatur auch in großen Kollektiven nur als Einzelfälle beschrieben wurde und ein komplexes ligamentäres Trauma mit funktioneller Trennung von Radiokarpal-, Intrakarpal- und Lunotriquetralgelenk impliziert (Taleisnik et al., Palmar carpal instability secondary to dislocation of scaphoid and lunate: report of a case and review of literatur. J Hand Surg 1982; 7: 606 -612). Die Lig. radiocarpale palmare und dorsale, das Lig. capitatoscaphoideum, die Gelenkkapsel des mediokarpalen Kompartimentes und das Lig. lunotriquetrum sind rupturiert. In Abhängigkeit vom Zustand des Lig. radio-scapholunatum können das Os lunatum und das Os scaphoideum als Einheit oder unabhängig von einander dislozieren. Eine Verletzung des Lig. radio-scapho-lunatum bedeutet dabei zwangsläufig auch eine Verschiebung der scapholunären Gelenkflächen.

Eine mögliche Erklärung für dieses Verletzungsmuster könnte eine progressive Dorsalextension des Handgelenkes sein, die zuerst zu einer perilunären Dislokation des Handgelenkes und anschließend zu einer palmaren Dislokation des Os lunatum führte. In dieser Position folgt das proximale Ende des Os scaphoideum dem Os lunatum. Bei zusätzlicher Ulnardeviation des Handgelenkes ist das Os scaphoideum dann nur noch gering von der distalen Konkavität des Radius abgedeckt und kann so nach palmar dislozieren (Gordon et al., Scaphoid and lunate dislocation. Report of a case in a patient with peripheral neuropathy. J Bone Joint Surg 1972; 54: 1769 - 1772).

Voraussetzung für eine funktionell komplette Ausheilung ist eine frühzeitige Reposition und Retention der Luxation. Die exakte Ausrichtung der Gelenkflächen kann postoperativ durch eine Dünnschicht-CT gesichert werden, die parallel zur Längsachse des Unterarmes ausgerichtet ist (Schmitt et al., Imaging and staging of avascular osteonecroses at the wrist and hand. Europ J Radiol 1997; 25: 92 -103). Eine gefürchtete Kompli- kation stellt die ischämische Nekrose der zum Teil von Endarterien versorgten Handwurzelknochen dar (Freedman et al., Vascularity of the carpus. Clin Orthop 2001; 383: 47 -59). Die Vitalität der Handwurzelknochen kann schon frühzeitig in einer Kontrastmittel-unterstützten MRT überprüft werden. Neben $\mathrm{T}_{1}$ - und $\mathrm{T}_{2}$-gewichteten Sequenzen wird die Durchführung fettunterdrückter Sequenzen empfohlen (Schmitt et al.). Im weiteren Krankheitsverlauf kann sich eine radiokarpale bzw. intrakarpale Instabilität einstellen, für die sich häufig schon in den konventionell-radiologischen Übersichtsaufnahmen Hinweise finden (Metz et al., Ligamentous instabilities of the wrist. Europ J Radiol 1997;
25: $104-111)$. Funktionsaufnahmen bzw. Schnittbilduntersuchungen können in diesen Fällen die Diagnose sichern. Karpale Instabilitäten werden chirurgisch mit transartikulären Bohrdrähten oder transfixierenden Platten bzw. Zugschrauben mit Spongiosaplastik und adaptierenden Nähten der identifizierbaren Bandstrukturen behandelt. In unserem Fall fand sich weder im klinischen noch im radiologischen Verlauf ein Hinweis auf eine karpale Instabilität. Der komplette Faustschluss war schon zwei Monate nach der Metallentfernung möglich.F. Schellhammer, J. Isenberg, H. G. Brochhagen, Köln 